\title{
PAPER
}

\section{Peripheral neuropathy in metachromatic leucodystrophy. A study of 40 cases from south India}

\author{
P S Bindu, A Mahadevan, A B Taly, R Christopher, N Gayathri, S K Shankar
}

J Neurol Neurosurg Psychiatry 2005;76:1698-1701. doi: 10.1136/jnnp.2005.063776

See end of article for authors' affiliations

Correspondence to: $\operatorname{Dr}$ A B Taly, Department of Neurology, National Institute of Mental Health and Neurosciences, Hosur Road, Bangalore 560 029, India; abtaly@yahoo.com

Received 18 January 2005 Revised version received 2 May 2005 Accepted 4 May 2005
Background: There is a paucity of literature from India on metachromatic leucodystrophy (MLD), a rare metabolic disorder of childhood resulting from aryl sulfatase A (ASA) deficiency.

Patients/Methods: Case records of histopathologically verified cases of MLD, evaluated over a period of 12 years at the National Institute of Mental Health and Neurosciences, Bangalore, India, were reviewed. Results: The late infantile group (36) manifested with regression of milestones (all), delayed mile stones (14), gait abnormalities (14), and seizures (11). Despite spasticity (29), there was hypo/areflexia in 25 patients. Optic atrophy (six) was rare. Consanguinity was noted in 25 children and four had a history of similar illness in siblings. Behavioural problems dominated in the juvenile group (four), but associated cognitive decline and hyporeflexia provided a clue to the diagnosis. Low serum ASA (seven of 20), raised cerebrospinal fluid protein (five of 12), and urinary metachromatic granules (two of 32) were infrequent. Electrophysiological evidence of severe demyelinating and length dependent sensory motor neuropathy was observed in all, even in the presence of hyper-reflexia. In addition to metachromatic dysmyelinating neuropathy in all patients, sural nerve biopsy in 20 patients revealed orthochromatic deposits within perivascular macrophages, particularly among those patients with normal ASA values (11 of 14), suggesting the accumulation of other glycosphingolipids.

Conclusions: This study produced some noteworthy observations: the high degree of consanguinity associated with MLD in India, the existence of MLD with normal serum concentrations of ASA, the deposition of orthochromatic lipids, and electrophysiological evidence of a partial conduction block.
$M$ etachromatic leucodystrophy (MLD) is a rare metabolic disorder of childhood caused by aryl sulfatase A deficiency. The accumulation of sulfatides occurs not only in the central nervous system, but also in various other tissues, including the peripheral nervous system. ${ }^{12}$

The association of peripheral neuropathy with MLD is known, but this fact is largely overlooked. ${ }^{3}$ Although the cardinal clinical manifestations are dominated by central nervous system involvement, signs of peripheral nervous system involvement are also evident on careful clinical examination. Our study highlights the importance of evaluating the peripheral nervous system in patients with suspected MLD.

\section{PATIENTS AND METHODS}

Our study involved 40 patients with MLD in whom the peripheral nerve revealed the presence of metachromatic material in frozen sections stained with cresyl violet. The case records of these patients were reviewed for clinical, electrophysiological, and pathological features. Patients older than 18 years and those in whom a diagnosis of MLD was suspected but the peripheral nerve failed to reveal metachromatic material were excluded.

Electrodiagnostic tests were performed using standard equipment and techniques. ${ }^{45}$ Conduction velocities in motor nerves were measured in median and peroneal nerves. Sensory nerve action potentials were elicited from median and sural nerves. For each nerve, the distal latency, conduction velocity, and amplitude were recorded.

Aryl sulfatase A was assayed using the artificial substrate, $p$-nitrocatechol sulfate $(\mathrm{n}=20){ }^{6}{ }^{6}$ The assessment of urine for metachromatic granules $(\mathrm{n}=32)$ and neuroimaging (computerised tomography, 22; magnetic resonance imaging, six) were also carried out.
A $2 \mathrm{~cm}$ length of the sural nerve was biopsied along the lateral melleolus and half of it was processed for paraffin wax embedding. The sections were routinely stained with haematoxylin and eosin, Kulchitsky Pal (for myelin), Maison's trichrome (for stromal elements), and monoclonal antibodies to neurofilaments to delineate the axons. A segment from the other half of the biopsy was cryosectioned and stained with cresyl violet to demonstrate the presence of metachromatic material. A limited number of samples were processed for araldite embedding and $1 \mu \mathrm{m}$ thick toluidine blue stained sections were studied to delineate the myelin pathology. Ultrathin sections stained with uranyl acetate and lead citrate were examined under Jeol 100CX at $60 \mathrm{kv}$.

\section{RESULTS}

The patients were divided into two groups based on age at onset of symptoms: (1) late infantile MLD with age at onset

Table 1 Clinical features of patients with late infantile metachromatic leucodystrophy $(n=36)$

\begin{tabular}{lll}
\hline Clinical features & N & $\%$ \\
\hline Delay in milestones* & 14 & 38.8 \\
Frequent falls† & 14 & 77.7 \\
Ataxia & 10 & 27.7 \\
Seizures & 11 & 30.5 \\
Bulbar symptoms & 12 & 33.3 \\
Spasticity & 29 & 80.5 \\
Sluggish stretch reflexes & 25 & 69.4 \\
Sluggish ankle jerks & 29 & 80.5 \\
Optic atrophy & 6 & 16.6 \\
\hline \multirow{2}{*}{ *Delayed milestones before the onset of regression; tamong those who } \\
attained independent walking.
\end{tabular}

Abbreviation: MLD, metachromatic leucodystrophy 


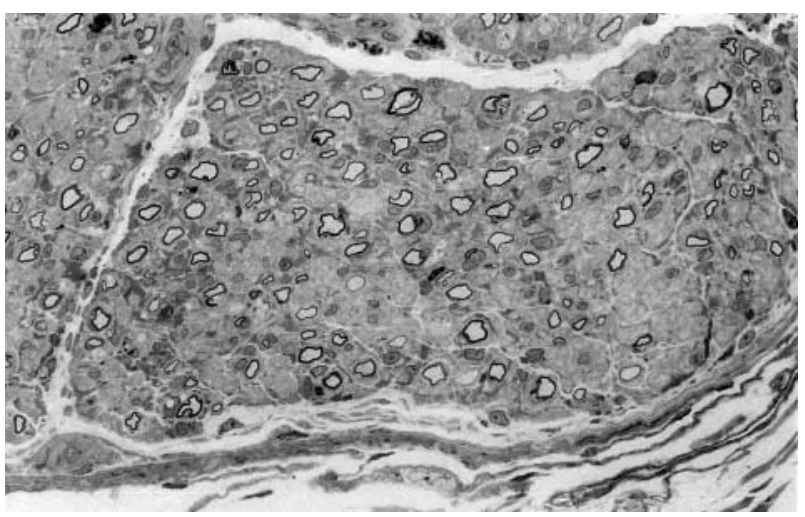

Figure 1 Diffuse hypomyelination involving both large and small diameter fibres. Toluidine blue stain; original magnification, $\times 240$.

between $\mathrm{l}$ and 4 years $(\mathrm{n}=36)$ and (2) juvenile MLD $(\mathrm{n}=4)$, with age at onset above 4 years.

In the late infantile group, the mean (SD) age of onset of the symptoms was 25.3 (11.3) months and the mean (SD) duration of the illness before presentation was 17.5 (12.2) months. Fourteen children had a baseline delay in milestones, followed by regression, whereas others developed regression after a period of normal development. Eighteen children attained independent walking before the onset of illness, and 14 of these children had frequent falls as the initial symptom. In two patients, one in the late infantile group and one in the juvenile group, the initial presentation was predominantly peripheral neuropathy, and the diagnosis of MLD was suspected when they subsequently presented with progressive loss of milestones. In four families there was one other affected sibling with a clinical history suggestive of MLD. One of the siblings was confirmed by the histopathological study of a peripheral nerve. Parental consanguinity was prominent in our study group, 25 patients being born to consanguineous parents. Table 1 summarises the clinical features in the late infantile group.

In the juvenile MLD group, all four patients presented with behavioural abnormalities such as attention deficits, hyperactivity, inappropriate laughter, and hyper-oral behaviour. They also had learning problems and scholastic backwardness. Three patients had myoclonic jerks and all the patients had sluggish or absent deep tendon reflexes, which indicated underlying neuropathy. The electrophysiological and histopathological features were essentially similar in both age groups.

Metachromatic granules in the urine were detected in two of 32 patients tested. Cerebrospinal fluid protein concentrations were raised in five of 12 patients and ranged between 76 and $124 \mathrm{mg} \%$ (mean, 84). Serum aryl sulfatase A estimation was carried out in 20 patients. The concentration was low in seven patients (normal, 35-135 mmol/litre) and within the normal range in 13 .

Computed tomography scans in 22 patients showed bilaterally symmetrical non-enhancing white matter hypodensities, consistent with a diagnosis of MLD. The areas involved were predominantly frontoparietal periventricular and deep white matter. In addition, magnetic resonance imaging in six patients showed sparing of subcortical " $U$ " fibres and better delineation of the involvement of corticospinal tracts and corpus callosum.

Motor conduction studies were carried out on the median nerve $(n=20)$ in the forearm segment and the common peroneal nerve $(n=29)$ in the leg segment. In all instances, the median nerves revealed a severe reduction in conduction velocity, with a mean $(\mathrm{SD})$ of 14.27 (4.7) $\mathrm{m} / \mathrm{s}$ (normal, $\geqslant 41$ ).
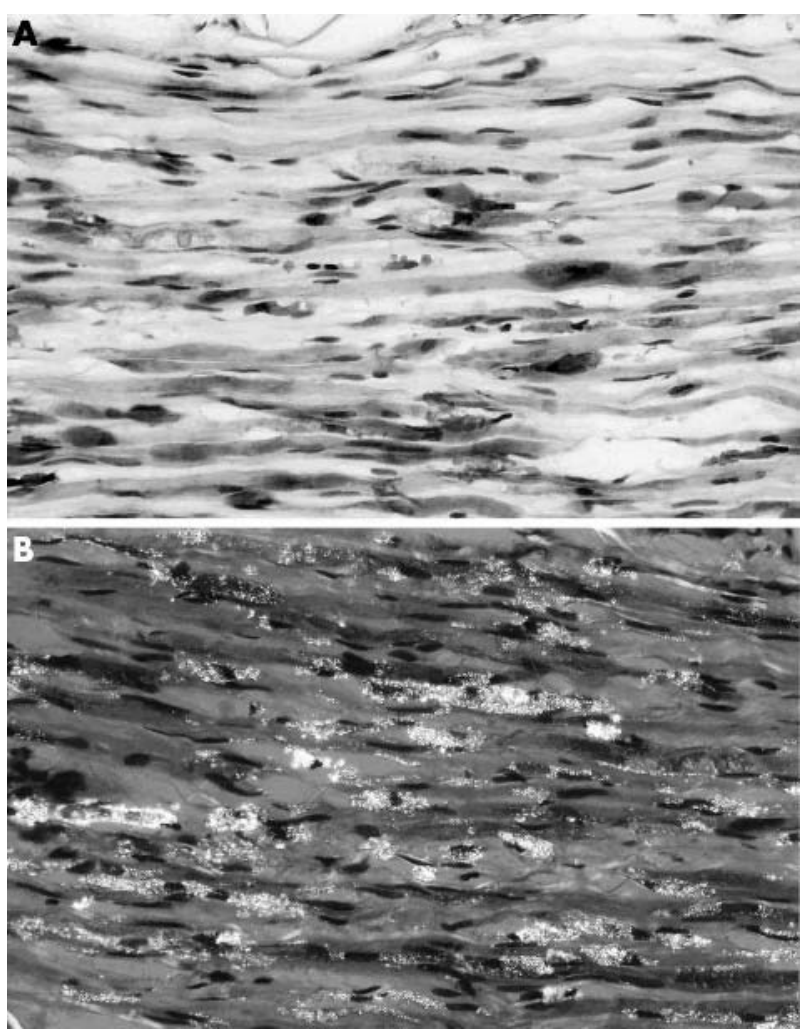

Figure 2 (A) Nerve biopsy stained with cresyl violet demonstrating dense metachromatic deposits within Schwann cells obscuring the nerve fibres in the endoneurium. Cresyl violet stain; original magnification, $\times 240$. (B) The metachromatic sulfatide material shows characteristic yellow green dichroism under crossed polarised light. Original magnification, $\times 240$.

In half of these patients the compound muscle action potential amplitude was less than the lower limit of normal (normal, $\geqslant 3.5 \mathrm{mV}$ ). The common peroneal nerve was unexcitable in two patients, whereas in the others the nerve conduction velocities were reduced (mean, $14.8 \mathrm{~m} / \mathrm{s} ; \mathrm{SD}$, 6.22 ; normal, $\geqslant 44$ ). The amplitude of the compound muscle action potential was reduced in 21 patients (normal, $\geqslant 2.5 \mathrm{mV}$ ). A partial conduction block, between the proximal and distal sites, was seen in two patients in the late infantile group and one in the juvenile group. ${ }^{7}$ This was present in the median nerve in two patients and both the median and common peroneal nerves in the third patient.

Sensory conduction parameters were evaluated in the median nerve using the orthodromic technique $(n=18)$. In 14 patients, sensory potentials could not be elicited, whereas in the remaining patients conduction velocities were moderately reduced (mean, $26.8 \mathrm{~m} / \mathrm{s} ; \mathrm{SD}, 7.3 ;$ normal, $\geqslant 55.3$ ). The sural nerve was assessed using the antidromic method $(\mathrm{n}=31)$. Sensory action potentials were absent in 25 patients and the conduction velocities were reduced in the remainder (mean, $21.18 \mathrm{~m} / \mathrm{s}$; SD, 8.9; normal, $\geqslant 45$ ).

On light microscopic examination of the biopsied sural nerves, diffuse, uniform hypomyelination with abnormal myelin configuration and remodelling were noted in all biopsies (fig 1). Bloated Schwann cells, containing granular metachromatic storage material (fig 2A), which revealed characteristic yellow green dichroism under crossed polarised light (fig 2B), were seen in all the nerves. In addition, active myelin degradation in the form of fine granular, osmiophillic debris in Schwann cells and macrophages was noted in 11 biopsies. This feature did not correlate with age at onset and duration or stage of the disease. Despite prominent Schwann 


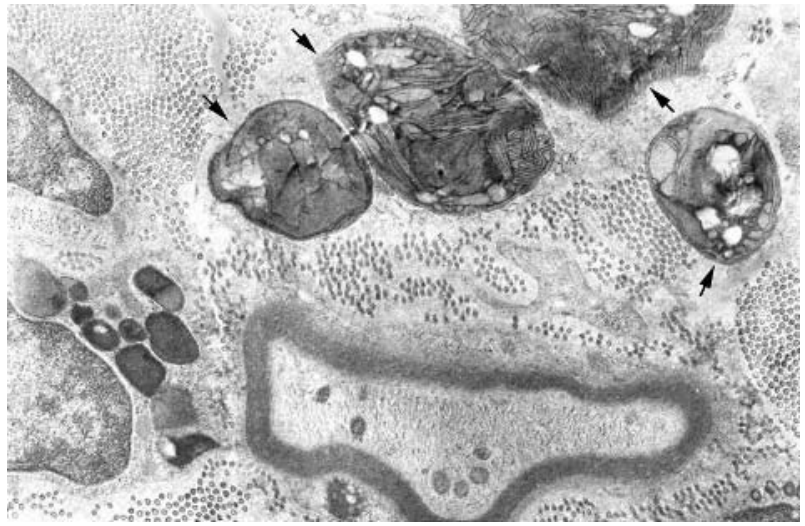

Figure 3 Electron micrograph showing tuff stone inclusion bodies composed of stacks of lamellar discs and plates (arrows) enclosed within a membrane in the endoneurium. Original magnification, $\times 10000$.

cell proliferation, onion bulb formation was conspicuously absent. The myelinated fibre population, both large and small, was reduced to varying degrees-severe in three, moderate in 28, and mild in five. Axonal stains revealed concomitant loss of both large and small diameter axons. The extent of axonal loss did not correspond with the duration of illness or the age at onset. Metachromatic storage material on cresyl violet staining was detectable within Schwann cells and endoneurial macrophages, and varied in amount and topographic distribution. On electron microscopic examination of the nerve biopsies, characteristic round to oval tuff stone inclusions were seen within the Schwann cell cytoplasm and free in the endoneurium among the collagen (fig 3). These inclusions had lamellar and prismatic discs arrayed in stacks enclosed within a thin membrane. Some of the inclusions were small and dense capped by lamellae, and these were seen as extracellular aggregates. No distinct zebra body types of inclusions were seen. The myelinated fibres revealed myelin breakdown and thinning and remodelling of the myelin sheath, reflecting the features seen on semithin sections. The axons revealed varying degrees of degeneration.

A unique finding seen in 20 biopsies was bright granular, eosinophillic, circumscribed deposits within endoneurial perivascular macrophages (fig 4) and occasional Schwann cells. Aryl sulfatase A values were available for 14 of the 20 patients with these findings. It is noteworthy that 11 of these

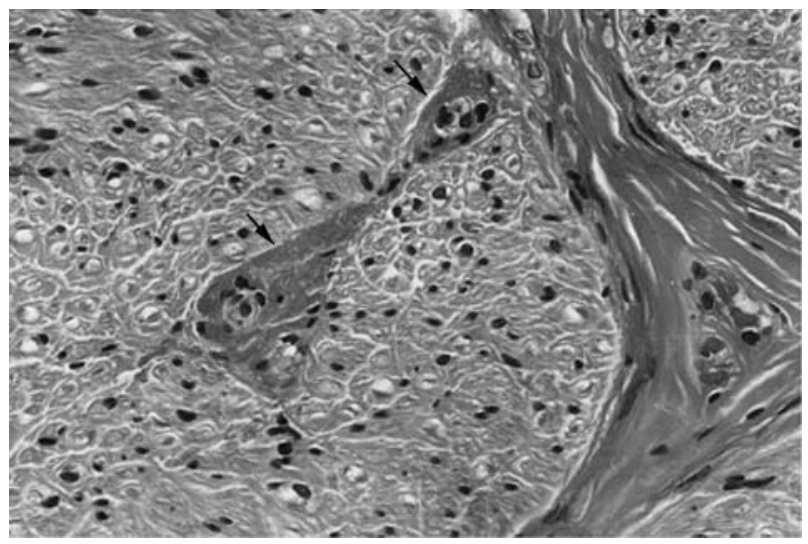

Figure 4 Accumulation of eosinophillic, orthochromatic material within perivascular histiocytes in the endoneurium (arrows) in cases of metachromatic leucodystrophy with normal aryl sulfatase A concentrations. Haematoxylin and eosin stain; original magnification, $\times 400$. patients had normal aryl sulfatase A concentrations. This material was osmiophillic, periodic acid Schiff positive, but non-metachromatic on cresyl violet staining, and resisted dissolution by the lipid solvents used in routine paraffin wax processing, suggesting that it is a form of glycoprotein conjugated with lipid. This adventitious material was noted in patients of both the late infantile and juvenile groups.

\section{DISCUSSION}

Our study involved 40 patients with histological evidence of metachromasia in sural nerve biopsies. In addition, electron microscopy on 10 biopsies revealed typical lamellar and prismatic tuff stone inclusions in the Schwann cell cytoplasm and in the endoneurium.

The role of sural nerve biopsy in the diagnosis of MLD has been amply stressed. ${ }^{89}$ Metachromasia in nerve biopsy is specific to MLD when appropriate staining techniques are used. Moreover, the yellow green dichroism seen under crossed polarised light is caused only by the sulfatide material found in MLD. ${ }^{10}$ Evidence of demyelination on electrophysiological studies and neuroimaging with clinical findings of central and peripheral nervous system involvement is common to many conditions included under the rubric of progressive cerebral degeneration in childhood. Hence, additional parameters such as the biochemical demonstration of aryl sulfatase A deficiency becomes necessary for establishing the diagnosis. In view of the pseudodeficiency of aryl sulphatase A seen in certain psychiatric and neurological disorders, the demonstration of metachromatic deposits in the tissues assumes paramount importance in discriminating MLD from other conditions, making tissue examination the most specific means of confirming the diagnosis. ${ }^{11}{ }^{12}$

Our patients were divided into late infantile and juvenile groups according to the age of onset. Twenty five children were born to consanguineous parents, with more than one sibling affected in four families, suggesting an autosomal recessive inheritance. Although the prevalence of marriages between close biological relatives is low in most Western countries it is as high as $33 \%$ in south India. ${ }^{13}$ In our study, consanguineous marriages were recorded in 25 of 40 parents, indicating the influence of consanguinity on the high incidence of recessive disorders.

Cardinal clinical features were characteristic gait abnormalities and recurrent falls with regression of milestones. ${ }^{14}{ }^{15}$ In our present series, a baseline delay in milestones was seen in 14 children and 15 patients never attained independent walking. All the patients with juvenile MLD presented predominantly with behavioural problems, but sluggish or absent muscle stretch reflexes provided a clue to the underlying neuropathy.

Raised cerebrospinal fluid protein in a child with progressive cerebral degeneration favours the diagnosis of MLD. It was noted in five of 12 patients, a lower frequency than described previously. ${ }^{14}$ The examination of urine for metachromatic granules was not rewarding, being positive in only two patients.

Serum aryl sulfatase A concentrations were within the normal range in 13 patients, despite evidence of accumulation of metachromatic material in the sural nerve. A variant of MLD has been described in which patients have normal concentrations of aryl sulfatase A but increased urinary sulfatide excretion, decreased catabolism of sulfatide by cultured fibroblasts following a loading dose, and evidence of sulfatide storage on sural nerve biopsy. ${ }^{16}$ We need to establish whether the patients in our study with normal aryl sulfatase A concentrations genuinely represent the $\mathrm{AB}$ variant or the sphingolipid activator protein deficiency. Despite these phenotypic differences in pathological features, there was no apparent difference in age at onset, clinical presentation, 
brain imaging, or electrophysiological parameters between patients with normal or low aryl sulfatase A values.

We observed length dependent neuropathy with reduced conduction velocity involving the sensory nerves more often than the motor nerves, similar to earlier reports. ${ }^{17-20}$ Conduction block, a hallmark of acquired neuropathies, has only rarely been reported in cases of MLD. ${ }^{21}$ This feature was noted in three children in our present study. Interestingly, two of them were siblings and in one case the nerve biopsy showed dense inflammatory infiltrates.

Two patients, one in the late infantile group and one in the juvenile group, presented predominantly with features of acute peripheral neuropathy and were diagnosed as having Guillain-Barré syndrome. The diagnosis of MLD was suspected later when they developed signs of mental regression. This presentation of MLD as a pure peripheral neuropathy simulating Guillain-Barré syndrome is rare. ${ }^{3} 1819$ 22-24

Sural nerve biopsy has proved to be the most appropriate method for the diagnosis of MLD. ${ }^{21}$ The electrophysiological alterations have paralleled the structural changes of segmental demyelination in nerve biopsy. ${ }^{125}$ An additional and yet distinct observation in our study was the presence of orthochromatic deposits in sural nerves mainly seen in patients with normal aryl sulfatase A values (11 of 14). This type of non-metachromatic, periodic acid Schiff positive material has previously been reported in a patient with activator protein deficiency. ${ }^{26}$ The normal aryl sulfatase A concentrations seen in most of the patients in our present study emphasises the diagnostic importance of electrophysiological studies and nerve biopsy in patients suspected to have MLD.

This is the largest cohort of MLD in the literature from a single centre in south India. Molecular genetic testing was not done because of the lack of facilities. Nevertheless, our study has provided some noteworthy observations, namely: the high consanguinity rate, the presence of a large subgroup with normal aryl sulfatase A concentrations, the partial conduction block seen in three patients, and the presence of orthochromatic deposits in addition to metachromatic material in the nervous tissue. Further studies to establish the higher prevalence of activator protein deficiency among south Indian patients with MLD are needed.

\footnotetext{
Authors' affiliations

P S Bindu, A B Taly, Department of Neurology, National Institute of Mental Health and Neurosciences, Hosur Road, Bangalore 560 029, India

A Mahadevan, N Gayathri, S K Shankar, Departments of Neuropathology, National Institute of Mental Health and Neurosciences, Bangalore

R Christopher, Department of Neurochemistry, National Institute of Mental Health and Neurosciences, Bangalore

Competing interests: none declared
}

\section{REFERENCES}

1 Webster HD. Schwann cell alterations in metachromatic leukodystrophy: preliminary phase and electron microscopic observations. J Neuropathol Exp Neurol 1962;21:534-54.

2 Bischoff A. Neuropathy in leukodystrophies. In: Dyck PJ, Thomas PK, Lambert EH, eds. Peripheral neuropathy. Philadelphia: WB Saunders, 1975:891-913

3 De Silva KL, Pearce J. Neuropathy of metachromatic leukodystrophy. J Neurol Neurosurg Psychiatry 1973;36:30-3.

4 Garmstorp I. Normal conduction velocity in ulnar, median and peroneal nerves in infancy, childhood and adolescence. Acta Paediatr 1963;146(suppl):68-76.

5 Gamstorp I, Shelburne SA. Peripheral sensory conduction in ulnar and median nerves of normal infants, children and adolescents. Acta Paediatr Scand 1965;4:309-14.

6 Baum H, Dogson KS, Spencer B. The assay of aryl sulfatases A and B in human urine. Clin Chim Acta 1950;4:453-5.

7 Onley RK. Consensus criteria for the diagnosis of partial conduction block. Muscle Nerve 1999;22(suppl):S225-9.

8 Vos AJ, Joosten EM, Gabreels FJ, et al. The diagnostic value of sural nerve biopsy in metachromatic leukodystrophy and other conditions with low aryl sulfatase activities. Neuropediatrics 1982;13:42-6.

9 Olsson Y, Sourander P. The reliability of the diagnosis of metachromatic leukodystrophy by peripheral nerve biopsy. Acta Pediatr Scand 1969;58:15-24.

10 Peiffer J. Metachromatic leukodystrophy. In: Vinken PJ, Bruyn GW, Moser HW, eds. Handbook of clinical neurology, leukodystrophies and poliodystrophies, Vol. 10. Amsterdam: Elsevier, 1970:43-62.

11 Kappler J, Watts RW, Conzelmann E, et al. Low arylsulfatase activity and choreoathetotic syndrome in three siblings: differentiation of pseudodeficiency from metachromatic leukodystrophy. Eur J Pediatr 1991;150:287-90.

12 Farrell K, Aplegarth DA, Toone JR, et al. Pseudoarylsulfatase-a deficiency in the neurologically impaired patient. Can J Neurol Sci 1985;12:274-7.

13 Bittles AH, Radha Ramadevi A, Savithri HS, et al. Consanguineous marriage and postnatal mortality in Karnataka, South India. Man New Series 1987;22:736-45.

14 MacFaul R, Cavanagh N, Lake BD, et al. Metachromatic leukodystrophy: review of 38 cases. Arch Dis Child 1982;57:168-75.

15 Zlotogora J, Costeff H, Elian E. Early motor development in metachromatic leukodystrophy. Arch Dis Child 1981;56:309-10.

16 Hahn AF, Gordon BA, Feleki V, et al. A variant form of metachromatic leukodystrophy without arylsulfatase deficiency. Ann Neurol 1982;12:33-6.

17 Miller RG, Gutmann L, Lewis RA, et al. Acquired versus familial demyelinative neuropathies in children. Muscle Nerve 1985;8:205-10.

18 Yudell A, Gomez MR, Lambert EH, et al. The neuropathy of sulfatide lipidosis (metachromatic leukodystrophy). Neurology 1967;17:103-11.

19 Aziz H, Pearce J. Peripheral neuropathy in metachromatic leukodystrophy. BMJ 1968;4:300.

20 Fullerton PM. Peripheral nerve conduction in metachromatic leukodystrophy (sulphatide lipidosis). J Neurol Neurosurg Psychiatry 1964;27:100-5.

21 Cameron CL, Kang PB, Burns TM, et al. Multifocal slowing of nerve conduction in metachromic leukodystrophy. Muscle Nerve 2004;29:531-6.

22 Taly AB, Santhosh V, Balamurugan N, et al. Remitting relapsing polyradiculoneuropathy in juvenile metachromatic leukodystrophy. Annals of the Indian Academy of Neurology 2004;7:543-8.

23 Tasker W, Chutorian AM. Chronic polyneuritis of childhood. J Pediatr 1969;74:699-708.

24 Martinez AC, Ferrer MT, Fucyo E, et al. Peripheral neuropathy detected on electrophysiological study as first manifestation of metachromatic leocodystrophy in infancy. J Neurol Neurosurg Psychiatry 1975;38:169-74.

25 Dayan AD. Peripheral neuropathy of metachromatic leukodystrophy: observations on segmental demyelination and remyelination and intracellular distribution of sulphatide. J Neurol Neurosurg Psychiatry 1967;30:311-14.

26 Schlote W, Harzer K, Christomanou H, et al. Sphingolipid activator protein deficiency in metachromatic leukodystrophy with normal arylsulfatase $A$ activity: a clinical, morphological, biochemical and immunological study. Eur J Pediatr 1991;150:584-91. 